\title{
Effect of Soil Properties on Safety Performance of W- beam Guardrail
}

\author{
Tso-Liang Teng ${ }^{1,}$, , Cho-Chung Liang ${ }^{2}$, Ching-Yu Hsu ${ }^{3}$, Chien-Jong Shih ${ }^{4}$ and Thanh-Tung Tran ${ }^{2}$ \\ ${ }^{1}$ Department of Mechanical Engineering, Hsiuping University of Science and Technology, Taichung, Taiwan \\ ${ }^{2}$ Department of Mechanical and Automation Engineering, Da-Yeh University, Changhua, Taiwan \\ ${ }^{3}$ Department of Marine Mechanical Engineering, ROC Naval Academy, Taiwan \\ ${ }^{4}$ Department of Mechanical and Electro-Mechanical Engineering, Tam-Kang University, Taipei, Tawan \\ *Corresponding author
}

\begin{abstract}
W-beam guardrails are the most widely used road safety barriers worldwide. They are used for protecting vehicle occupants on dangerous areas of roadways. The ability of $\mathrm{W}$ beam guardrails to absorb some of the crash energy reduces the risk of injury for vehicle occupants and limits deformation of impacting vehicles. Once the rail separates from the posts, the rail forms a redirection ribbon, guiding the vehicles away from the nearby hazard. Thus, the soil-post interaction in W-beam guardrails play a critical role in the manner in which errant vehicles interact with safety barriers. All road safety barriers used on European highways are designed according to the European standard EN 1317. This study applied the finite element code LS-DYNA for evaluating the safety performance of W-beam guardrail when the post rammed into various soil properties. Four crash test simulations were conducted for evaluating the crashworthiness of the $W$-beam guardrail according to European Standard EN 1317. The results have demonstrated that the soil properties does not affect the impact severity (ASI, THIV) but affects working width.
\end{abstract}

Keywords-crashworthiness; EN1317; LS-DYNA; W-beam guardrail

\section{INTRODUCTION}

Road safety barriers are roadside structures that are installed on certain sections of road to improve highway safety by preventing a vehicle from leaving the road and colliding with roadside hazards. Road safety barrier systems used on European highways must fulfil the European standard EN 1317 [1]. The European standard EN 1317 provides criteria for determining the levels of vehicle containment, appropriately redirecting errant vehicles to the road, and providing guidance for pedestrians and other road users. Safety barriers are typically designed according to EN1317 by using three main criteria: vehicle containment, impact severity, and barrier working width.

The W-beam guardrail is the most widely used road safety barrier worldwide. It is used for protecting specific locations on the road that comprise dangerous zones and areas. Conventionally, W-beam guardrails comprise a rail element (called a W-beam) and supporting posts (Figure 1).

The ability of W-beam guardrails to absorb some of the crash energy reduces the risk of injury for vehicle occupants and limits deformation of impacting vehicles. Upon impact, the posts absorb some of the crash energy by rotating in the surrounding soil before separating from the rail as they undergo full yield. Once the rail separates from the posts, the rail forms a redirection ribbon, guiding the vehicles away from the nearby hazard. Thus, the soil-post interaction in W-beam guardrails play a critical role in the manner in which errant vehicles interact with safety barriers. Therefore, understanding the effect of the soil on safety performance is imperative.

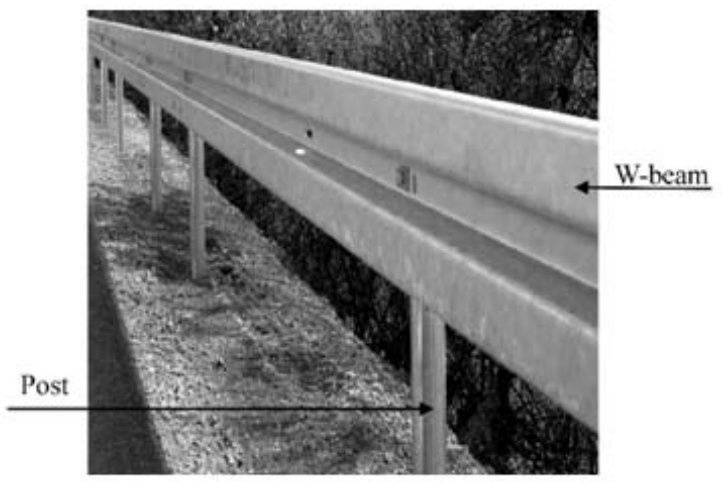

FIGURE I. W-BEAM GUARDRAIL [8]

The current study applied the finite element code LSDYNA for evaluating the safety performance of W-beam guardrail when the post rammed into various soil properties. This study presents crashworthiness test results for W-beam guardrails with various soil condition according to the European standard EN 1317.

\section{EUROPEAN STANDARD EN 1317}

The European standard EN 1317 was approved by the European Committee for Standardization in March 1998 and was revised on April 29, 2010 [1]. This standard provides guidelines for evaluating real impact tests on roadside barriers. The standard prescribes criteria that road safety barriers must fulfill to reduce the severity of accidents related to roadside barriers.

Safety barriers were designed according to the European standard EN 1317 by evaluating three main criteria for various performance levels: containment level, the acceleration severity index (ASI) and theoretical head impact velocity 
(THIV). To ensure safety, these indicators must not exceed the determined limits (Table 1).

TABLE I. IMPACT SEVERITY LEVELS

\begin{tabular}{|c|c|c|}
\hline Impact severity level & Index values & \\
\hline A & $\mathrm{ASI} \leq 1.0$ & \\
$\mathrm{~B}$ & $1.0<\mathrm{ASI} \leq 1.4$ & $\mathrm{THIV} \leq 33 \mathrm{~km} / \mathrm{h}$ \\
$\mathrm{C}$ & $1.4<\mathrm{ASI} \leq 1.9$ & \\
\hline
\end{tabular}

Barrier deformation is expressed according to the working width $\left(\mathrm{W}_{\mathrm{m}}\right)$, which is the maximum lateral distance between any part of the barrier on the undeformed traffic side and the maximum dynamic position of that part of the barrier. The deformation of road safety barriers can be categorized into eight classes (W1-W8).

\section{FINITE ELEMENT MODEL OF ROAD SAFETY BARRIER IMPACT TEST}

\section{A. Road safety Barrier Model}

AG04-2.0 A-type barrier was used for this study, and this barrier was produced by the ALKA group according to the European standard for road safety barriers [2]. Figure 2 shows the simulation models of the $\mathrm{W}$-beam guardrail system.

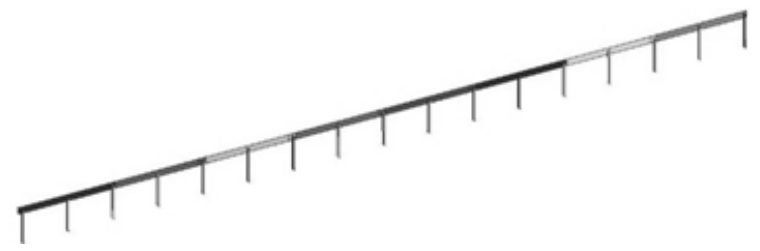

FIGURE II. W-BEAM GUARDRAIL MODEL

The system comprised W-shaped guardrails and C-posts. The length of the W-beam guardrail segments was $4,300 \mathrm{~m}$. The C-post was $1,600 \mathrm{~mm}$ in length and was embedded 950 $\mathrm{mm}$ in the soil. The dimensions of the post were $125 \mathrm{~mm} \times$ $62.5 \mathrm{~mm} \times 25 \mathrm{~mm}$. The distance between each post was $2 \mathrm{~m}$. The height of the barrier was $750 \mathrm{~mm}$ from the ground.

All safety barrier parts were modeled using fully integrated shell elements (having an average size of $20 \mathrm{~mm}$ ) with five integration points throughout the shell thickness for preventing a zero-energy mode (hourglass mode) [3],[4]. The bolt connection between the $\mathrm{W}$-shaped segments and the posts were modeled using a spot-weld element. The W-beam guardrail component materials, such as the posts and W-beam, were represented using MAT024 (a piecewise linear plasticity material model) in LS-DYNA.

\section{B. Vehicle Model}

A Geo Metro vehicle model (version GM-R3) available in the NCAC database [5] was used in this simulation. The vehicle model was developed and improved in Politecnico di
Milano, Italy and is publicly accessible on the NCAC Web page.

\section{Soil Modelling}

The soil was modeled according to a subgrade modulus approach and processed as laterally loaded piles. The subgrade modulus is influenced by many factors (e.g., properties of the soil and width of the posts) and has no standard value. This study followed the method proposed by Habibagahi and Langer [6] and Plaxico et al. [7] that was based on the bearing capacity approach for basic soil.

The post-soil interaction was modeled using nonlinear directions on the two adjacent sides of the posts because they work both tension and compression. On one side, these springs attached all the nodes of the cross-section and on the other side, the node was constrained in all six directions. Nine-layer springs with a spacing of $100 \mathrm{~mm}$ was used presented post-soil interaction. The stiffness of the nonlinear springs increased with depth and was determined on the basis of the aforementioned equations.

\section{Boundary Condition}

The impact test model comprised the vehicle and safety barrier. The vehicle speed was set to $100 \mathrm{~km} / \mathrm{h}$ with an impact angle of $20^{\circ}$ according to the TB11 test regulation. Elastic springs were added at both ends of each node along the depth of the W-beam to represent guardrail's continuation.

\section{ANALYSIS OF COMPUTATIONAL RESUlt}

Figure 3 show the impact test results of the road safety barrier finite element models. In all four cases, the barriers prevented the vehicle from leaving the road and redirected it onto the road.
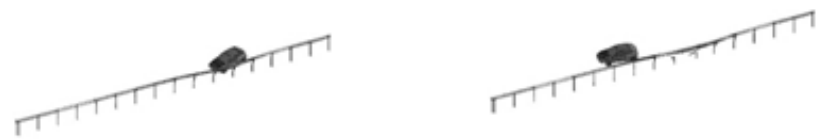

(A) LOOSE SAND
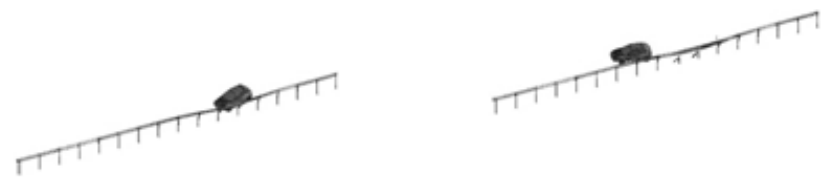

(B) MEDIUM SAND
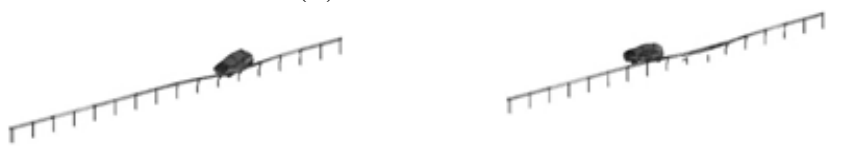

(C) DENSE SAND
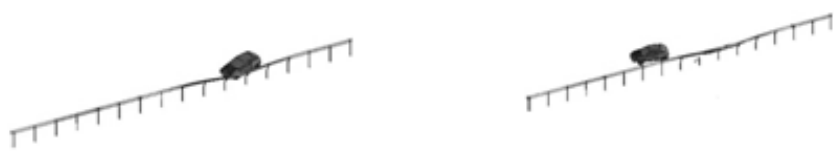

(D) VERY DENSE SAND

FIGURE III. SEQUENTIAL FROM SIMULATION TEST AT 0.1S (LEFT) AND 0.5S (RIGHT) WITH VARIOUS SOIL PROPERTIES 
TABLE II. SIMULATION RESULTS WITH VARIOUS SOIL CONDITION

\begin{tabular}{|c|c|c|c|}
\hline Soil type & ASI & THIV (km/h) & $\begin{array}{c}\text { Working width } \\
(\mathbf{m m})\end{array}$ \\
\hline Loose sand & 0.93 & 26.1 & 805 \\
\hline Medium sand & 0.96 & 27.2 & 940 \\
\hline Dense sand & 0.96 & 26.8 & 1040 \\
\hline Very dense sand & 0.95 & 25.7 & 1300 \\
\hline
\end{tabular}

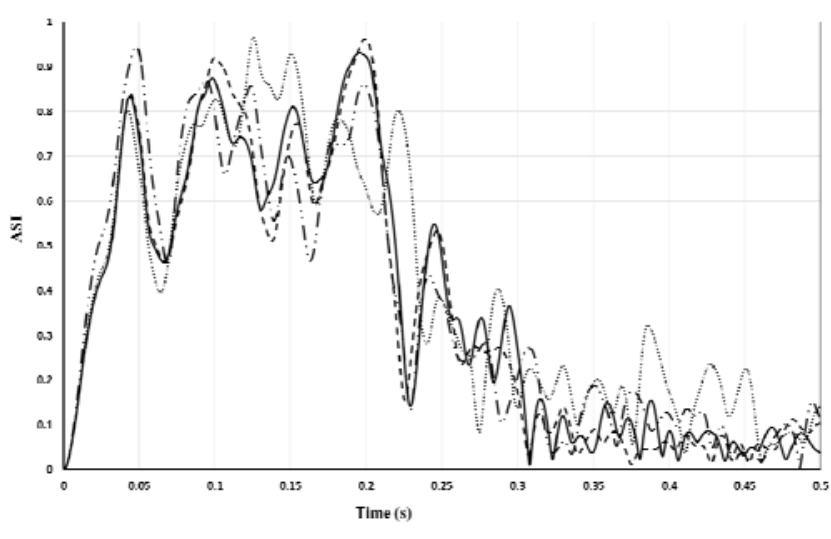

FIGURE IV. ACCERLERATION SEVERITY INDEX VALUE OVER TIME WITH VARIOUS SOIL CONDITION

Table 2 show the impact severity (ASI and THIV) and working width of the simulation models test results of the road safety barrier finite element models. In all four cases, the barriers meet the EN1317-2 requirement with impact severity corresponded to class A. A very slight difference was observed in the ASI and THIV value while the difference between working width values are higher. The result have clearly indicated that the soil properties doesn't not effect to impact severities (ASI, THIV). Figure 4 illustrates the ASI values obtained over time for the three barriers with various soil condition. Figure 5 showed the deflection of the four barriers with various soil condition. The results indicated that the barrier working width increase with the stiff of soil. The barrier showed the highest working width (i.e., $1300 \mathrm{~mm}$ ) when the post rammed into the very dense sand soil, and the barrier demonstrated the lowest working width (i.e., $805 \mathrm{~mm}$ ) when the post rammed into the loose sand soil. Figure 5 showed the deflection of the four barriers with various soil condition. The results indicated that the barrier working width increase with the stiff of soil. The barrier showed the highest working width (i.e., $1300 \mathrm{~mm}$ ) when the post rammed into the very dense sand soil, and the barrier demonstrated the lowest working width (i.e., $805 \mathrm{~mm}$ ) when the post rammed into the loose sand soil.

\section{CONCLUSION}

The study applied the finite element code LS-DYNA to evaluate the safety performance of $\mathrm{W}$-beam guardrail, which had various soil condition, when impacted by a $900-\mathrm{kg}$ small passenger car (TB11 test). Four crash test simulations were conducted to evaluate the crashworthiness of the W-beam guardrail at various soil properties according to the European standard EN1317. The results have demonstrated that the soil properties does not effect the impact severity ( ASI, THIV) but effects working width.

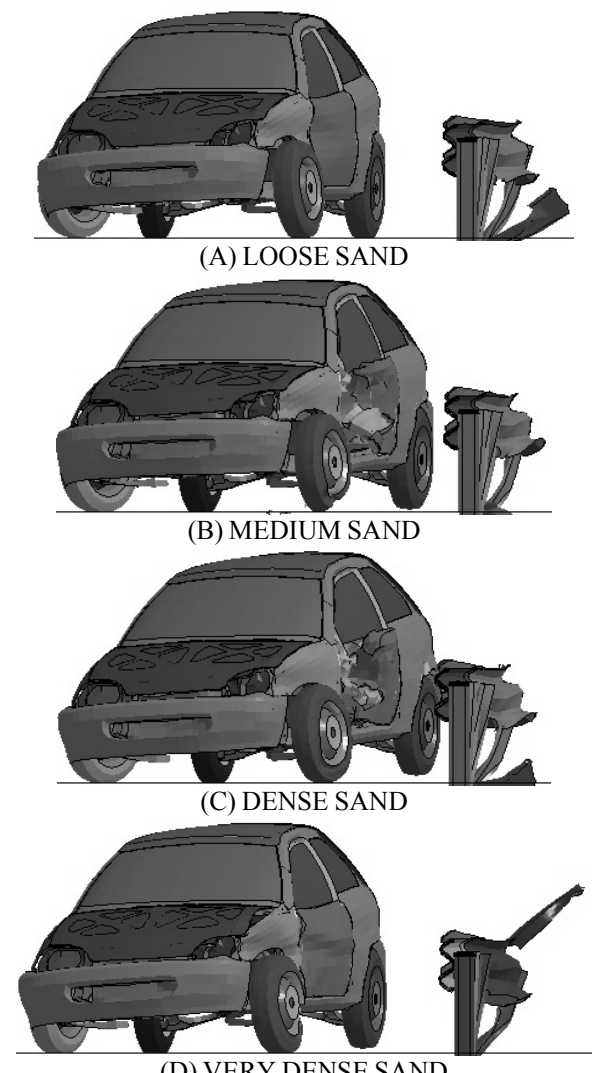

FIGURE V. PERFORMANCE OF ROAD SAFETY BARRIER AND VEHICLE AT $0.5 \mathrm{~S}$

\section{REFERENCES}

[1] European Committee for Standardization, European Standard EN 1317-1, EN 1317-2, Road Restraint Systems, 2010.

[2] Ali Osman Atahan, Ayhan Oner Yucel, Muhanmet Musab Erdem. Crash testing and evaluation of a new generation L1 containment level guardrail. Engineering Failure Analysis. 2014 (38) 25-37.

[3] LS-DYNA Keyword user's manual, Livermore Software Technology Corporation, 2007.

[4] LS-DYNA Theoretical Manual, Livermore Software Technology Corporation, Livermore 2006.

[5] Finite Element Model Archive, FHWA/ NHTSA National Crash Analysis Center. 2008. Http://Www.ncac.gwu.edu/vml/models.html.

[6] K. Habibagahi and J. A. Langer. Horizontal Subgrade Modulus of Granular Soils, Laterally Loaded Deep Foundations. American Society for Testing Materials, 1984, 21-34.

[7] C. A. Plaxico, G. S., Patzner and M. H. Ray. Finite element modelling of guardrail timber posts and the post-soil interaction. Transportation Research Record 1647, 1998, 139-146.

[8] Safe German Guardrail Technology, Heintzmann Basic Barrier. http://www. heintzmann.eu 